\title{
The Digital protection and Inheritance Research of Intangible Cultural Heritage against the Background of "Internet Plus"
}

\author{
Jiajue Fang ${ }^{1 . a}$, Hong Yu ${ }^{1 . b}$, Shanyuan $\mathrm{Ge}^{1 . \mathrm{c}}$, Zhihui Mo ${ }^{1 . d .}{ }^{*}$ \\ ${ }^{1}$ Guangxi Science \& Technology Normal University, Laibin, China 546199 \\ a jiayu10000@163.com, b1129242853@qq.com, c839853292@qq.com, d37388757@qq.com \\ ${ }^{*}$ Corresponding author
}

Key words: Internet plus; intangible cultural heritage; digital protection; inheritance

\begin{abstract}
The advent of the time of "Internet Plus" has triggered the profound changes in many social dimensions, which has brought the opportunity of innovative development to culture and offered technological support to the manner of the protection and inheritance of intangible cultural heritage (hereinafter called ICH). By analyzing the current research on the protection and inheritance of ICH conducted by related scholars, the article has proposed the promotion of the protection and inheritance of ICH from the perspective of "Internet Plus".
\end{abstract}

\section{Introduction}

On March 5, 2015, for the first time, the government report delivered by Premier Li Keqiang at the $3^{\text {rd }}$ Session of the 12th National People's Congress mentioned the "Internet Plus" action plan. "Internet Plus" is called a new state of utilizing the platform of the Internet as well as information and communication technology to combine the Internet with various walks of life including traditional industries. "Internet Plus" contains not only the energetic drive at technological level but also a prominent cultural creativity. Therefore, the extension of "Internet Plus" to the field of ICH has brought a brand new opportunity for the protection, inheritance, innovation and industrialized development of ICH. By taking advantage of the national strategic momentum, we should launch a comprehensive campaign to make the culture of ICH "alive". On the basis of this situation, it will be an effective cultural development path to construct the digital protection and inheritance mode under the background of "Internet Plus".

2. The development Trend of the Digital protection and Inheritance of ICH at Home and Abroad

Since the 1990s, with the application of information technology in various walks of life, "digitalization" has stealthily and swiftly entered into the research field of cultural heritage and ICH. As a result, the explorative focus of disciplines such as museology, library science and archival science was placed on the digitalization of cultural relics, ancient books and archives, thus facilitating the onset of the researches such as digital archaeology, digital preservation and digital management. In terms of technological level, by way of the collection, storage, processing, demonstration and dissemination of digital resources, the resources of ICH are transformed, reproduced and restored to the digital form that is shared by the world and continuously regenerated .

In 2003, in UNESCO's Convention for the Safeguarding of the Intangible Cultural Heritage, the concept of ICH was officially defined. In Intangible Cultural Heritage Law of the People's Republic of China promulgated in 2011, the term "ICH" shall refer to various traditional cultural manifestations which are handed down by the people of all nationalities from generation to generation and regarded as part of their cultural heritage, and objects and spaces relevant to traditional cultural manifestations. The state shall preserve the ICH by determination, recording, filing, etc.

The development of information technology has provided the protection of ICH with effective 
technological support. Thereupon, experts and scholars at home and abroad have begun to focus their research on information form of ICH. For example, countries such as Italy, Japan and Canada have conducted various digitalized projects of $\mathrm{ICH}$. The countries that carried out an earlier research on the digitalization of ICH include France, ROK and Japan. However, in the past decade, countries including China, Italy and India have conducted some related researches, which is fairly eye-catching. In terms of word frequency, the more frequent words on the research of ICH contain ontology, preservation, digital preservation, multimedia, digital archives, metadata, semantic net, etc. . The hotspots about the research on the digitalization of ICH abroad are classified as three levels such as ICH digitalization collection, ICH digitalization preservation and ICH digitalization. In terms of ICH digitalization collection, more attention is paid to the themes such as the compilation, recording and filing about the list of $\mathrm{ICH}$. In terms of ICH digitalization preservation, attention is focused on the research of ontology and metadata. As to the research on the development of ICH digitalization, topics such as augmented reality, virtual reality technology and database construction are mainly mentioned ${ }^{[5]}$. Domestically, the research topics about the digital protection and inheritance mainly focus on the connotation and necessity research on ICH digital protection as well as method and path research. Judging from research fields, regional and ethnic ICH digital protection research plays a major part. In fact, a wide range of disciplines are involved in the research on the application of ICH digitalization technology. According to CNKI (China National Knowledge Internet)'s statistics in the category of disciplines, the achievement in this regard involve 41 disciplines such as computer software and its application, library \& information and digital library, archives and museums, news and media, fine arts, calligraphy, sculpture and photography, physical education, music and dancing and ethnology. According to the query through CNKI, from 2005 when the first article entitled Shandong University of Art and Design Has Made Great Efforts to Deal with the Digital protection of ICH was published to 2015, more than 30 articles were published every year. Starting from different discipline characteristics, experts from various discipline fields conducted the research on the digital protection of ICH from different perspectives, which inevitably facilitated the development of the digitalization of ICH.

\section{The Opportunity Brought to ICH by Technology in "Internet Plus" Time}

Under the background of informatization, the changes of information resource management and the means of ICH digital protection brought by technology have aroused the attention and research on the part of public cultural institutions such as libraries, archives, museums and science \& technology museums as well as academic research institutions and protection institutions of ICH. Although various institutions, due to the difference of their own scopes of business, put particular emphasis on the kind of ICH digital protection that they are interested in, their ultimate goal is, through the application of information technology including Internet technology, big data technology, cloud technology, VR and AR technology, to optimize the filing, preservation, inheritance, utilization and dissemination of $\mathrm{ICH}$ protection and conduct a more scientific reorganization, research, propaganda and demonstration of ICH so as to ensure the vitality of ICH. In the process of advancing toward its large-scale expansion, the construction of ICH digitalization resource database, in a large amount of ICH digital information resources that are heterogeneous and from multiple sources, is faced with the issue of how to conduct classification, preservation, management and dissemination in a scientific and effective way. In actual work, in most cases, it is designated departments or institutions that complete the construction including the process from the compilation of ICH list to the recording and filing of $\mathrm{ICH}, \mathrm{ICH}$ database and website, thus leading to the decentralization of the specific implementation of ICH digital protection. At the same time, internationally, the research topics of ICH digital protection are also relatively decentralized. Judging from the aspect of technology application, some research institutions are engaged in the research on information classifying organizations, filing mechanisms and standards as well as the development of ICH information retrieval while others take relevant policies, mechanisms and systems as their research topics. 


\section{Construct the Pattern of the Digital protection and Living Inheritance of "Internet Plus ICH"}

Figure 1. Pattern of digital protection and living inheritance of "Internet Plus ICH"

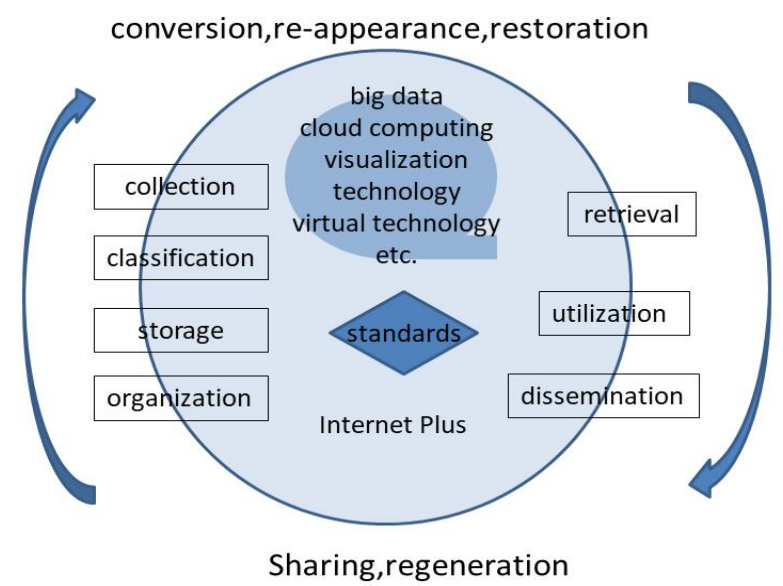

At present, the ICH digital information is characterized by decentralized sources and complicated or even fragmented structure. In this context, it can provide a living development vitality for the digitalization inheritance of ICH to make use of the new network technology of "Internet Plus" platform and construct the scientific pattern of ICH digital information resource featuring collection, classification, storage, organization, retrieval, utilization and dissemination, as shown in Figure 1.

\subsection{Accelerate the construction of the standard norm of digital protection}

The project collection and digital protection of $\mathrm{ICH}$ constitute the cardinal work as well as a priority of ICH protection. In recent years, the related standards based on the ten classifications of the representative project list of national level ICH have offered a theoretical basis and operation guide to the digital protection of ICH in our country. In November 2017, sponsored by the Department of Culture \& Technology under the Ministry of Culture, the National Culture and Art Resource Standardization Technology Committee held the expert reasoning conference of recommendatory trade standards in the series of the digital protection of ICH. Some related standards will be officially put forward, which will provide professional reference and technological guidance for national ICH protection institutions to carry out the work of the digital protection of ICH.

\subsection{Give play to the new technology in optimizing the work of digitalized collection to improve the quality and utilization of digitalized resource}

Digital protection of ICH is a systematic project. Through the process concerning the digital resource of ICH including collection, production, storage, organization, management, development, utilization and dissemination, the restoration, reappearance and demonstration of ICH itself as well as the sharing, reuse and dissemination of $\mathrm{ICH}$ digital information are realized. Under the background of Internet Plus, as a representative information technology, "big data" can be summarized as four "V"s, namely, volume, variety, velocity and value. And the process procedure of big data is characterized by collecting data information, utilizing distributed technology to conduct the query and classification of massive data and mine data to satisfy the demand of data analysis. With ICH information resource processed by big data, it is likely to rapidly obtain the result of statistical analysis of many kinds of information so as to greatly improve the pertinence and effectiveness of the protection work of ICH.

The real presentation of the physical spaces such as existential space and demonstration museum concerning ICH is the urgent but difficult problem in the digital protection of ICH. Is it feasible to bring in the blue-chip 360-degree panoramic technology to solve this problem? Based on static images, 360-degree panoramic technology can realize virtual reality technology on the micro-computer platform. It helps people to get a 360-degree panoramic observation on the Internet. In addition, through interactive manipulation, free browse can be realized so as to display a three-dimensional VR visual world. This technology can make people have a look at the 
architectural features of various cultural relics and surroundings panoramically without going out. It can make people shelter themselves in the living context of $\mathrm{ICH}$, upgrade their sensory perception and produce emotional resonance. The application of 360-degree panoramic technology to the digitalization of ICH can not only improve work efficiency but also reduce costing so as to promote the protection work of $\mathrm{ICH}$.

\subsection{Cloud technology is used to construct the database resource platform of ICH}

The application of digital technology to the protection and inheritance of ICH is embodied in the following four aspects. Firstly, digital collection and storage technology has provided the guarantee for the complete protection of ICH; secondly, digital management and production technology has supplied a support for the effective inheritance of $\mathrm{ICH}$; thirdly, digital demonstration and dissemination technology has offered the platform to the wide sharing of ICH; fourthly, digital consumption technology has given the space to the development of ICH industrialization .

At present, our country is in the process of setting up the digital network resource platform and database of ICH step by step. In 2006, www.chinaich.mobi was opened. By applying digital technology and network platform to showcase and disseminate the professional knowledge of human ICH in China and even in the world, the website has fully displayed the profound and abundant ICH resources in China and forged the space for information exchange of the protection work of ICH which can be participated in by everyone, thus boosting the advancement of the protection work of ICH. Under this influence, related local websites have been opened in succession, including www.ichshanghai.cn and www.jsfybh.com and the like. Local museums boast not only their websites, mirco-blogs and Wechat, but also their digital virtual museums. In addition, their own official online stores have been opened on their own network shopping platforms in order to popularize their derivative cultural and creative products. Now, established local ICH databases are faced with problems such as unevenness in contents, scarcity in information and difficulty in the connectivity and sharing of data. Fortunately, the cloud computing which is becoming more and more sophisticated serves as a good solution to data sharing. By "cloud computing", it is a pattern of the increase, utilization and delivery of related service based on the Internet, which usually provides resources that are dynamic, easy to expand and, in most cases, virtualized. Cloud is a metaphor of network and internet. The application of cloud technology places the database platform of ICH on the cloud computing platform of enterprises so as to enhance the synergy of the digital protection work of $\mathrm{ICH}$.

\subsection{Comprehensively utilize the function of publicity and development on the part of mobile media}

By the digital protection of $\mathrm{ICH}$, it is, on the basis of fully mastering new technology and by combining the status quo of development and practical problems of the ICH in China, the realization of the diversified application of resources through the protection, publicity, inheritance and utilization of ICH. Nowadays, people are highly dependent on mobile media in aspects such as acquisition of information, sharing and exchange as well as shopping. In the time when people cannot live without mobile phone, it is obvious that new media have become a very effective instrument of propaganda. It is of highly pivotal use to make the best of the advantage of new media to upgrade the influence and living space of ICH. Therefore, it is necessary to design and develop the mobile applications of ICH projects characterized by diversified forms, rich contents and different functions and open WeChat Official Accounts. By releasing kinds of news, introductions and videos related to $\mathrm{ICH}$, relevant content information should be timely issued and upgraded and various activities such as voting and giving awards should be launched in good time so as to form the propaganda and demonstration system featuring clear classification, rich contents and diverse means. For example, in Liuzhou of Guangxi, the public Wechat platform was utilized to disseminate the related items about the folk activities of ethnic minorities on the third day of the third lunar month in 2017, which pushed the ICH activity to the transmission platform at any time to make the masses to know about the living ICH closely related to their daily life, thus effectively boosting the popularization and influence of the ICH activity and making ICH enjoy more popular support. In 
the photo exhibition of Guangxi International Ethnography held by Guangxi Museum of Nationalities, the official QQ, official Wechat, official website and official Tencent Video Channel were set up. Of them, as of February 2017, the video view of Tencent surpassed 300,000 times . That is to say, new media technology is of great significance to the protection and inheritance of ICH.

ICH is the source of traditional Chinese culture and the root of Chinese nation. It contains abundant spiritual connotation and has bountiful historical and cultural value. The protection of ICH means the inheritance of the bloodline of traditional national culture. In the tide of "Internet Plus", with information technology as a sharp instrument, the creation of the strong body of science and technology application combining the inheritance and protection of ICH makes the inheritance and protection of $\mathrm{ICH}$ get further extension and derivation. In the era of the Internet, digitalization has provided a new approach and new opportunity for the protection of ICH. It has supplied ICH with more national characteristics to make ICH get perpetual inheritance and development.

With the unremitting and painstaking efforts on the part of the workers engaged in the protection of ICH, the theoretical system of ICH protection with Chinese characteristics has gradually come into being. The protection and inheritance of ICH extends in horizontal and vertical dimensions. On the one hand, it is necessary to constantly explore new thought and new approaches to meet the demand of national development strategy. On the other hand, attention should be paid to the complicated situation arising from cultural conflicts. Moreover, great efforts should be made to promote the ICH in our country to play a better role in sustaining the diversity of human culture and enhancing the mutual understanding, mutual respect and mutual appreciation among the peoples in the world in the process of participating in the protection of international $\mathrm{ICH}$.

\section{Acknowledgement}

This article is supported by the key research base of Humanities and Social Sciences in Guangxi Universities_-Yao Culture Research Base of Dayao Mountain in Jinxiu County of Guangxi. It is one of the research achievements of the study on the inheritance and development of the ethnic culture of the Guangxi Universities of science and technology, the "national culture strong area" of Guangxi.

\section{References :}

[1] Quan Xi."Internet Plus": Construct the New Scene of the Digitalized Inheritance of ICH [J]. Journal of Northwest Minzu University, 2017(6): 181

[2] Wang Yaoxi. The Digitalization of ICH[M]. Beijing: People's Publishing House,2009:18

[3] Zhao Yue, Zhou Yaolin. A Review of the International Research on Digital Preservation of Intangible Cultural Heritage [J]. Library, 2017(8):59-68

[4] Zhou Ya, Xu Xin. A Research Review on the Digitization of Intangible Cultural Heritage [J]. Library and Information Service, 2017(1): 6-13

[5] Huang Yonglin, Tan Guoxin, The Digital protection and Development Research on ICH in China [J].Journal of Huazhong Normal University (Humanities and Social Sciences), 2012 (2)

[6] Sun Chuanming, Cheng Qiang, Tan Guoxin. Current Situation and Countermeasure Analysis for Digital Protection of Intangible Cultural Heritage in Minority Areas of Guangxi [J]. Study of Ethnics in Guangxi, 2017(3):127

[7] Luo Wei, Wu Hao, Han Zehua. Research Report on the Development of the Protection of ICH in China in 2014 [J]. Arts Criticism, 2015(4): 41

[8] Zheng Jiqin. A Study on the Application of New Media Technology in Digital Protection and Spreading of Traditional Drama-Take Zhejiang Wuju Opera as Example [D].Zhejiang Normal University, 2016. 
[9] Tan Yiling. Study and Design Practice on Digital Protection in Gaeml Brocade Intangible Culture Heritage [D]. Hunan University, 2016.

[10]Cao Yaping. Research on Platform Design Based on Digital Protection of Intangible Cultural Heritage [D]. East China University of Science and Technology, 2016.

[11]Liu Canjiao, Yang Lixin. A Research Review on Digital Protection for Intangible Cultural Heritage in China [J]. Library,2016,02:15-20.

[12]Pei Zhanglong. Thinking on the Digital Protection of ICH in the New Situation of IT [J]. Art Science and Technology,2016,2902:43.

[13]Long Hu, Li Na. A Study on the Construction and Digital Protection of the ICH Inheritance System in the Southeast of Guizhou in the Era of Big Data [J]. Journal of Kaili University,2016 (12): :90-92. 